\title{
High-level C-X-C chemokine receptor type 4 expression correlates with brain-specific metastasis following complete resection of non-small cell lung cancer
}

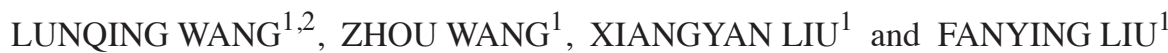 \\ ${ }^{1}$ Department of Thoracic Surgery, Provincial Hospital Affiliated to Shandong Univeristy, Jinan, Shandong 250021; \\ ${ }^{2}$ Department of Thoracic Surgery, Qingdao Municipal Hospital, Qingdao, Shandong 266071, P.R. China
}

Received August 4, 2013; Accepted February 18, 2014

DOI: $10.3892 / \mathrm{ol} .2014 .1979$

\begin{abstract}
Brain-specific metastasis is one of the primary causes of recurrence following complete resection of non-small cell lung cancer (NSCLC) and the underlying mechanism remains unclear. The present study was designed to investigate the correlation between $\mathrm{C}-\mathrm{X}-\mathrm{C}$ chemokine receptor type 4 (CXCR4) expression and brain-specific metastasis of NSCLC. Lung cancer tissues from 105 patients who underwent complete tumor resection between January 1998 and June 2008 (sample group, 34 with brain metastasis during the follow-up period; control group 1, 34 without metastasis during the follow-up period; and control group 2, 37 with other organ metastasis, excluding brain metastasis, during the follow-up period) were examined by immunohistochemistry to detect the expression of CXCR4 protein. The differences in CXCR4 expression were compared using McNemar's $\chi^{2}$ test. Estimation of survival was calculated with the Kaplan-Meier method and the statistical differences were analyzed with the log-rank test. Overexpression of CXCR4 protein was observed in $31(91.2 \%)$ NSCLC patients with brain metastasis, which was greater than that observed in the NSCLC patients with other organ metastases $(73.0 \% ; \mathrm{P}=0.048)$ and without metastases $(14.7 \%$; $\mathrm{P}<0.001)$. CXCR4 protein was highly overexpressed in patients with brain-specific metastasis, which indicated that high-level CXCR4 expression correlates with brain-specific metastasis of NSCLC.
\end{abstract}

\section{Introduction}

Surgery is the preferred treatment for non-small cell lung cancer (NSCLC). However, without satisfactory surgical results, the overall five-year survival rate is $\sim 30-40 \%$. The

Correspondence to: Dr Zhou Wang, Department of Thoracic Surgery, Provincial Hospital Affiliated to Shandong University, 324 Jingwu Weiqi Road, Jinan, Shandong 250021, P.R. China

E-mail:wz620226@hotmail.com

Key words: C-X-C chemokine receptor type 4, non-small cell lung cancer, metastases, brain majority of patients succumb following surgery due to tumor metastasis and recurrence. The brain metastases-associated clinicopathological features and molecular biological markers are clinically significant as they may determine a more accurate prognosis for the patient, allowing postoperative adjuvant therapies to be targeted. However, there is currently no effective method for identifying high-risk NSCLC patients with brain-specific metastasis.

Brain-specific metastasis is one of the primary causes of recurrence following complete resection of NSCLC, and the underlying mechanism remains unclear. The present study was designed to investigate the correlation between $\mathrm{C}-\mathrm{X}-\mathrm{C}$ chemokine receptor type 4 (CXCR4) expression and brain-specific metastasis of NSCLC.

The homing theory states that tumor cell metastasize to specific organs, due to an organ-specific capacity to arrest or attract specific types of cancer cells via chemotaxis (1). Therefore, the spreading of certain tumors is considered to be selective, rather than random This hypothesis is further affirmed by the observations of organ-specific metastasis in certain cancer types, such as prostate cancer, where the tumor cells are more likely to metastasize to bone (2). Previous studies have identified that CXCR4 and its ligand, CXCL12, are associated with organ-specific metastasis (2-4). Our previous preliminary study demonstrated that CXCR4 overexpression in tumor tissue is correlated with NSCLC at the homeochronous and heterochronic phases of solitary brain metastasis (5).

Based on previous studies, the present study retrospectively investigated NSCLC patients with brain metastasis following complete resection. Immunohistochemical methods were used to detect the expression of CXCR4 within tumor tissues. Through matched-pair analysis the correlation between CXCR4 overexpression and brain metastasis of postoperative NSCLC patients was investigated. The function of CXCR4 in brain-specific metastasis of NSCLC, according to the controlled analysis of patients with brain-specific metastasis and patients with other organ metastases, was also examined.

\section{Patients and methods}

Patients. Between January 1998 and June 2008, 5,117 patients underwent surgical resection of NSCLC at the Qingdao 
Municipal Hospital (Qingdao, China). A total of 105 (2.1\%) patients who underwent complete tumor resection were retrospectively reviewed. The patients in the present study did not receive any neoadjuvant therapy prior to surgery. This study was approved by the ethics committee of Qingdao Municipal Hospital (Qingdao, China). Patients provided written informed consent.

The sample group included 34 NSCLC patients with brain-specific metastasis during the follow-up period, which consisted of 22 males and 12 females, with a median age of 56 years (range, 37-75 years). Histological analysis revealed that there were 14 squamous cell carcinomas, 17 adenocarcinomas and three large cell lung cancers. According to the 2009 International Association for the Study of Lung Cancer tumor, node, metastasis (TNM) classification, seven patients were classified as Stage I, 11 patients were classified as Stage II and 16 patients were classified as Stage III. A total of 30 patients received 4-6 cycles of cisplatin-based chemotherapy (Table I).

Follow-up and diagnosis of metastases. A complete patient follow-up was performed on a regular basis. Every 3-6 months, the patients received comprehensive medical examinations as follows: Brain and thorax X-ray computed tomography (CT) scans; upper abdomen enhanced CT scans; and/or liver, gallbladder, pancreas, spleen, kidney and adrenal B-mode ultrasound; electroconvulsive therapy scans in the patients presenting with bone pain; and whole body PET-CT in certain patients. The newly identified lesions in the brain and other organs were diagnosed as metastases after eliminating the possibility of benign lesions. The pathological investigation following surgical resection confirmed brain-specific metastasis in 16 patients and the remaining patients were clinically diagnosed with brain-specific metastasis.

Research design and statistical analysis. A total of 34 eligible NSCLC patients without metastases during the follow-up period served as control group 1 (Table I) and 37 NSCLC patients with other organ metastases, excluding brain-specific metastasis, during the follow-up period served as control group 2 (Table II). All of these patients were screened in the following order: TNM stage, histological tumor type, degree of tumor differentiation, gender, surgical pattern, adjuvant therapy post-surgery and age. When these clinicopathological characteristics did not exactly match with the experimental group, the maximum extent of consistency principle was followed.

Pearson's $\chi^{2}$ test was used to evaluate differences in CXCR4 expression between the patients with brain-specific metastasis and the patients without hematogenous metastases; this enabled examination of the correlation between CXCR4 expression and postoperative brain-specific metastasis of NSCLC. The patients who received complete tumor resection at the same period as the other two groups and exhibited other organ metastases during the follow-up period were selected for control group 2. The $\chi^{2}$ test was used to evaluate differences in CXCR4 expression between brain-specific metastasis patients and other organ metastases patients in order to further examine the role of CXCR4 in postoperative brain-specific metastasis of NSCLC.
Table I. Clinicopathological features of patients with brain-specific metastasis and patients in control group 1 .

\begin{tabular}{|c|c|c|}
\hline $\begin{array}{l}\text { Clinicopathological } \\
\text { feature }\end{array}$ & $\begin{array}{c}\text { Brain } \\
\text { metastasis } \\
\text { (no. of cases) }\end{array}$ & $\begin{array}{c}\text { Control } \\
\text { group } 1 \\
\text { (no. of cases) }\end{array}$ \\
\hline
\end{tabular}

Age (years)

$<40$

$40-50$

$50-60$

$60-70$

$>70$

Gender

Male/Female

Surgical pattern

R. upper lobe

R. lower lobe

R. middle lobe

R. middle and lower lobes

R. whole lung

L. upper lobe

L. lower lobe

L. whole lung

Pathological type

Adenocarcinoma

Squamous cell carcinoma

Large cell carcinoma

Degree of differentiation

Well differentiated

Poorly differentiated

Undifferentiated

T status

T1/T2/T3/T4

$\mathrm{N}$ status

N0/N1/N2

$8 / 17 / 9$

Post-operative

adjuvant chemotherapy

Yes/No

$30 / 4$

$31 / 3$

Diagnosis of brain metastases

Pathology/Imaging

Transfer time from surgery

$<6$ months

$16 / 18$

0

6-12 months

2

12-24 months

6

24-36 months

12

$>36$ months

5

$\mathrm{R}$, right; L, left.

The clinical and pathological data were compiled into an SPSS 15.0 database (SPSS, Inc., Chicago, IL, USA). An estimation of survival rate was calculated using the Kaplan-Meier method and statistical differences were analyzed with the 
Table II. Clinicopathological features of patients with other organ metastases in control group 2.

\begin{tabular}{|c|c|c|c|c|c|}
\hline \multirow[b]{2}{*}{ Clinicopathological features } & \multicolumn{5}{|c|}{ Location of metastases $^{\mathrm{a}}$} \\
\hline & $\begin{array}{l}\text { Lung and pleura } \\
\qquad(\mathrm{n}=14)\end{array}$ & $\begin{array}{l}\text { Liver } \\
(\mathrm{n}=10)\end{array}$ & $\begin{array}{l}\text { Bone } \\
(\mathrm{n}=6)\end{array}$ & $\begin{array}{l}\text { Adrenal glands } \\
\qquad(\mathrm{n}=5)\end{array}$ & $\begin{array}{l}\text { Other } \\
(n=2)\end{array}$ \\
\hline \multicolumn{6}{|l|}{ Surgical pattern } \\
\hline Lobe/Whole lung & $12 / 2$ & $9 / 1$ & $6 / 0$ & $5 / 0$ & $1 / 1$ \\
\hline \multicolumn{6}{|l|}{ Pathological type } \\
\hline Adenocarcinoma & 7 & 5 & 2 & 1 & 0 \\
\hline Squamous cell carcinoma & 5 & 5 & 4 & 3 & 2 \\
\hline Large cell carcinoma & 2 & 0 & 0 & 1 & 0 \\
\hline \multicolumn{6}{|l|}{ Degree of defferentiation } \\
\hline Well differentiated & 5 & 6 & 3 & 2 & 2 \\
\hline Poorly differentiated & 7 & 3 & 3 & 3 & - \\
\hline Undifferentiated & 2 & 1 & 0 & 0 & 0 \\
\hline \multicolumn{6}{|l|}{ TNM stage } \\
\hline I & 2 & 1 & 0 & 1 & 0 \\
\hline II & 5 & 4 & 2 & 1 & 2 \\
\hline III & 7 & 5 & 4 & 3 & 0 \\
\hline \multicolumn{6}{|l|}{ Diagnosis of metastases } \\
\hline Pathology/Imaging & $6 / 8$ & $1 / 9$ & $1 / 5$ & $3 / 2$ & $2 / 0$ \\
\hline
\end{tabular}

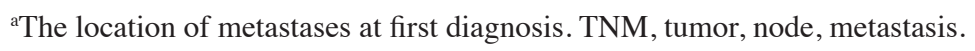

log-rank test. $\mathrm{P}<0.05$ was considered to indicate a statistically significant difference.

Techniques. Immunohistochemistry (streptavidin-peroxidase [SP] method) was performed to detect the CXCR4 expression levels in every tissue specimen. The primary antibody employed was the mouse anti-human CXCR4 monoclonal antibody at 1:100 dilution (Abcam Ltd.). Secondary processing of the tissue samples was performed with an SP kit and a universal secondary antibody kit according to the manufacturer's instructions (Beijing Zhongshan Golden Bridge Biotechnology Co., Ltd., Beijing, China). Briefly, following incubation overnight with the primary antibody, the secondary biotinylated antibody and subsequent avidin-biotin complex reagent were incubated for $30 \mathrm{~min}$, respectively. Staining was visualized using diaminobenzidine.

Paraffin-embedded specimens of the surgically removed tumor tissue were collected and all of the stained sections were examined by two independent experienced pathologists who had been double blinded to the clinical data. The immunohistochemical score was calculated by combining the proportion score (percentage of positive stained cells) with the staining intensity score. The proportion score ranged from 0 to 4 , as follows: 0 (no staining), 1 (1-24\%), 2 (25-49\%), 3 (50-74\%), $4(>75 \%)$. The staining intensity was scored as follows: 0 (negative), 1 (weak), 2 (moderate) and 3 (strong). The proportion score and staining intensities score were subsequently multiplied to generate the total IHS score for each case. The total score ranging 4-12 was considered to be positive expression. The tissue specimens were scored according to a combination
Table III. Comparison of CXCR4 expression in non-small cell lung cancer patients.

\begin{tabular}{lccc}
\hline & \multicolumn{3}{c}{ Expression of CXCR4 } \\
\cline { 2 - 4 } Location of metastases & Positive & Negative & Total \\
\hline Brain & 31 & 3 & 34 \\
Other organ & 27 & 10 & 37 \\
No metastases & 5 & 29 & 34 \\
\hline
\end{tabular}

CXCR4, C-X-C chemokine receptor 4.

of the intensity and the proportion of positive-stained tumor cells, as follows: i) The number of positive tumor cells was determined by assesment of 10 high-power fields of dense tumor cell areas, which were randomly selected for counting. A minimum of 600 tumor cells were counted and observed and the proportion of the positive-stained tumor cells was evaluated according to the following scale: $0=0-5 \% ; 1=6-10 \%$; $2=11-50 \%$; and $3 \geq 50 \%$. ii) The staining intensity was evaluated according to the following scale: $0=$ No reactivity; $1=$ low; $2=$ moderate; and 3=strong. A total score of 3-6 was considered to indicate positive staining of CXCR4 (Fig. 1) (6).

\section{Results}

The difference in CXCR4 protein expression between patients with brain-specific metastasis and the control groups. In 

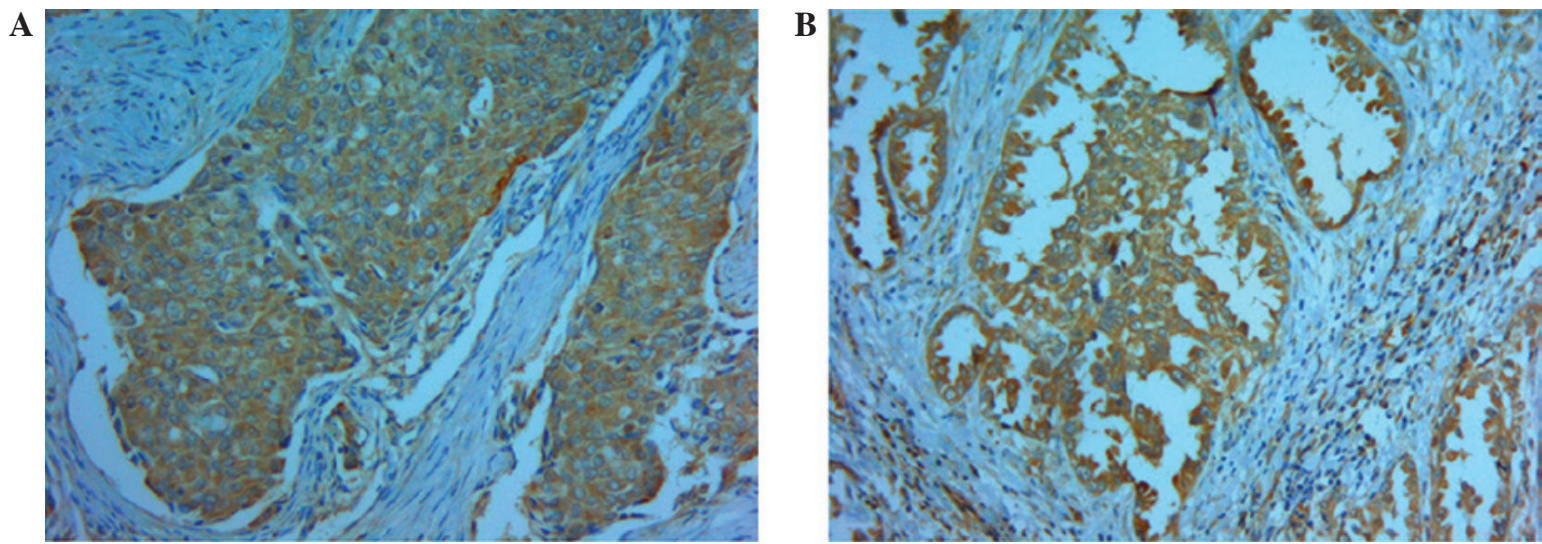

Figure 1. C-X-C chemokine receptor type 4 expression in non-small cell lung cancer. (A) Lung squamous cell carcinoma. (B) Lung adenocarcinoma. (Magnification, x200). Streptavidin peroxidase immunohistochemical staining was used for visualisation.

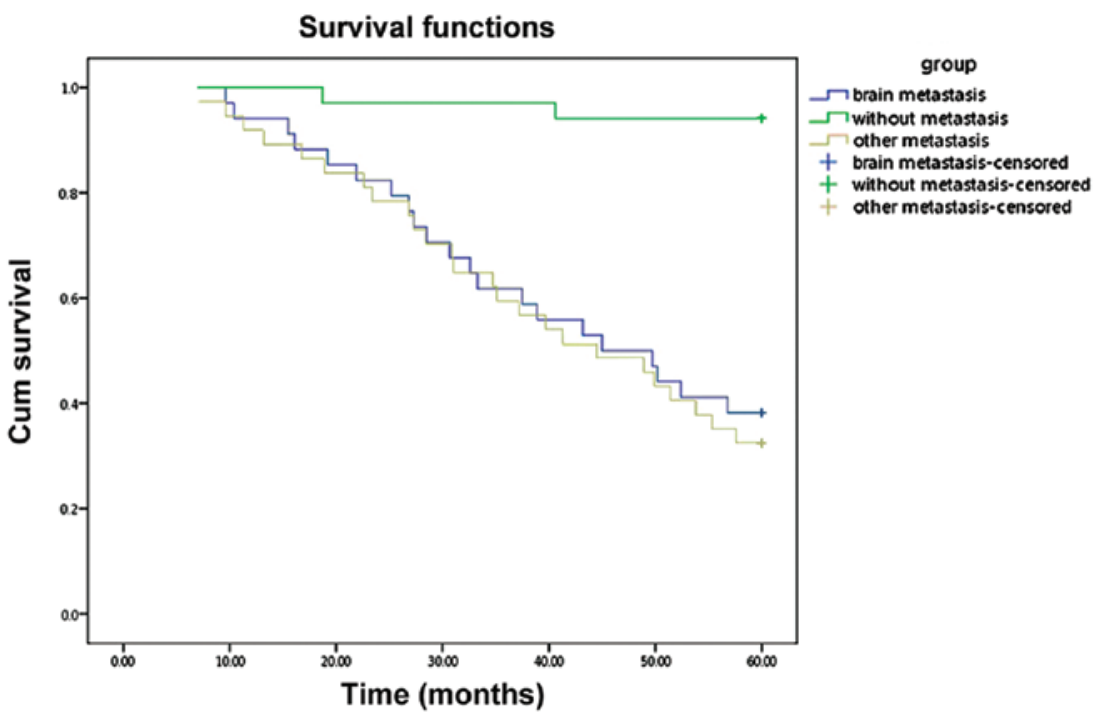

Figure 2. Kaplan-Meier curve of five-year cumulative survival rate.

the group of patients with brain-specific metastasis, CXCR4 overexpression was observed in 31 samples of NSCLC tissue (91.2\%), whereas of the 34 patients without metastases, CXCR4 overexpression was observed in five samples of NSCLC tissue (14.7\%), which was significantly lower compared with that of patients with brain-specific metastasis according to the $\chi^{2}$ tests $(\mathrm{P}<0.001$; Table III)

In the group of patients with other organ metastases, CXCR4 overexpression was observed in 27 samples of NSCLC tissue $(73.0 \%)$, which was significantly higher compared with that of patients without metastases $(14.7 \%$; $\mathrm{P}<0.001$; Table III).

The CXCR4 expression levels of the brain-specific metastasis group and other organ metastases group (control group 2) were compared. According to the $\chi^{2}$ test (Pearson method) the brain-specific metastasis patients exhibited a higher expression of CXCR4 (Table III), which was identified to be a statistically significant difference $(\mathrm{P}=0.048)$.

Analysis of survival rate among NSCLC patients with and without metastases. All patients with lung cancer were closely followed-up, with a mean follow-up time of 59.6 months (range, 7-116 months). The three- and five-year cumulative survival rates of 34 patients with brain-specific metastasis were 61.8 and $38.2 \%$, respectively and the three- and five-year cumulative survival rates of patients with other organ metastases were 59.5 and $32.4 \%$, respectively. The log-rank test demonstrated that there were no statistically significant differences identified between the survival rates of the two groups ( $\mathrm{P}>0.05$; Fig. 2$)$. The three- and five-year cumulative survival rates of the 34 patients without metastases were 97.1 and $94.1 \%$, respectively.

\section{Discussion}

Clinicopathological feature analyses have revealed that, for NSCLC patients presenting with brain-specific metastasis alone, metastatic lesion resection or other adjuvant therapies may improve quality of life and prolong survival time (7-9). Therefore, identifying patients with a high risk of brain-specific metastasis following lung cancer resection is clinically significant for predicting the prognosis and selecting the most appropriate adjuvant therapy.

Currently, the mechanism(s) that promote brain-specific metastasis have not been clearly elucidated. When NSCLC attacks the pulmonary vein, tumor cells are able to directly 
enter the blood circulation without pulmonary capillary bed involvement. Hematogenous spread may lead to multiple organ metastases, with the brain being the most common metastatic site clinically. Further studies are required to determine why NSCLC cells remain in the brain by hematogenous metastases and the underlying mechanisms regarding the specific affinity of NSCLC cells to the brain.

In recent years, the homing theory has been proposed as a result of investigations into tumor metastases to specific organs. The theory states that different organs chemotactically capture or attract particular types of tumor cells, which is termed homing and results in metastasis to specific organs (1). In 2001, Müller et al (3) proposed that tumor cells metastasize by a specific combination of chemotactic factors (chemokines) and receptors (chemokine receptor) to specific organs. It was identified that breast cancer cells express CXCR4 highly and that the ligand of CXCR4, CXCL12, is primarily expressed in the lungs, liver and bone marrow. It is also these same organs that the majority of breast cancer cells often metastasize to, providing strong correlational evidence in support of the homing theory. Clinically, NSCLC most commonly metastasizes to the brain. Whether this is also due to an interaction between chemotactic factors and their receptors requires further investigation.

In our previous study, the correlation of CXCR4 and solitary brain-specific metastasis at the homeochronous and heterochronic phases was examined. The preliminary results demonstrated that CXCR4 expression levels in the tumor tissue of NSCLC patients with brain-specific metastasis is higher compared with NSCLC patients without distant metastases (5). This indicated that CXCR4 may be associated with brain-specific metastasis in NSCLC. In order to further examine the correlation between CXCR4 overexpression and brain-specific metastasis following NSCLC surgical resection and to assess whether it is associated with brain-specific metastasis, the present study examined more cases. In addition, a comparison between patients with brain-specific metastasis and patients with other organ metastases was performed.

In the group of patients with brain-specific metastasis, CXCR4 overexpression was observed in 31 of the patients with brain-specific metastasis and, of the 34 patients without metastases, CXCR4 overexpression was observed in only five patients, which was a statistically significant difference. In the group of patients exhibiting other organ metastases, CXCR4 over-expression in tumor tissue was also higher compared with the patients without metastases. The present study indicates that CXCR4 overexpression in NSCLC may be correlated with postoperative hematogenous metastases. Further analysis by comparing patients exhibiting brain-specific metastasis and other organ metastases revealed that $\mathrm{CXCR} 4$ overexpression is higher in patients with brain-specific metastasis compared with patients exhibiting other organ metastases $(\mathrm{P}=0.048$; Table III). It was also demonstrated that a chemotaxis function may mediate the homing of CXCR4-overexpressing NSCLC cells to the brain, where the ligand CXCL12 is overexpressed.

Adopting statistical methods of matching comparison reduced the experimental bias and enhanced the objectivity of the present study. However, as a retrospective study, several limitations remain, including: i) Following surgery, the adjuvant chemotherapy scheme and medication-use time were not tightly controlled between the patients; ii) the majority of organ metastases patients were clinically diagnosed; iii) the number of cases involved in this single-center study was limited; iv) due to ethical considerations, it was not possible to obtain normal brain tissue for the detection of normal CXCL12 expression levels. However, the fact that CXCL12 is constitutively expressed in the developing and mature central nervous system $(10,11)$ may support the results of the present study indirectly. Previous studies have demonstrated that CXCL12 expression was normally controlled at a relatively low level (12-17). Under certain pathological situations, including HIV 1-associated dementia, brain tumor, ischemia and neuroinflammation, CXCL12 expression may be briefly upregulated. Astrocytes and vascular endothelial cells in the parenchyma have been proposed as two primary cell sources for inducible CXCL12, and hypoxia-inducible factor-1 (18) may regulate CXCL12 gene expression in endothelial cells, resulting in the selective expression of CXCL12. Furthermore, interleukin-1 $\beta$ (16) induces CXCL12 in astrocytes by extracellular signal-regulated kinase and phosphatidylinositol-4,5-bisphosphate 3-kinase signaling pathways. In the present study, although the majority of organ metastases occurred within three years postoperatively and all of the patients in the control groups had regular followup, no brain-specific metastasis was identified; however, the possibility that brain-specific metastasis may have occurred subsequently may not be ruled out. These problems should be addressed in future studies.

In conclusion, brain-specific metastasis is one of the primary reasons for recurrence following complete resection of NSCLC. At present, it is only possible to assess the occurrence of brain-specific metastasis according to clinicopathological features, which are considered to lack sensitivity. The present study demonstrated that CXCR4 overexpression in patients with brain-specific metastasis was higher when compared with the control group patients, indicating that the CXCL12/CXCR4 signaling axis may be involved in the brain-specific metastasis processes of NSCLC. Therefore, further studies are required to examine whether CXCR4 may be a molecular marker in predicting brain-specific metastasis associated with NSCLC.

\section{References}

1. Takeuchi H, Kitago M and Hoon DS: Effects of chemokines on tumor metastasis. Cancer Treat Res 135: 177-184, 2007.

2. Taichman RS, Cooper C, Keller ET, Pienta KJ, Taichman NS and McCauley LK: Use of the stromal cell-derived factor-1/CXCR4 pathway in prostate cancer metastasis to bone. Cancer Res 62: 1832-1837, 2002.

3. Müller A, Homey B, Soto H, Ge N, Catron D, Buchanan ME, et al: Involvement of chemokine receptors in breast cancer metastases. Nature 410: 50-56, 2001.

4. Phillips RJ, Burdick MD, Lutz M, Belperio JA, Keane MP and Strieter RM: The stromal derived factor-1/CXCL12-CXC chemokine receptor 4 biological axis in non-small cell lung cancer metastases. Am J Respir Crit Care Med 167: 1676-1686, 2003.

5. Chen G, Wang Z, Liu XY and Liu FY: High-level CXCR4 expression correlates with brain-specific metastases of non-small cell lung cancer. World J Surg 35: 56-61, 2011.

6. Spano JP, Andre F, Morat L, Sabatier L, Besse B, Combadiere C, et al: Chemokine receptor CXCR4 and early-stage non-small cell lung cancer: pattern of expression and correlation with outcome. Ann Oncol 15: 613-617, 2004.

7. Koutras AK, Marangos M, Kourelis T, Partheni M, Dougenis D, Iconomou G, et al: Surgical management of cerebral metastases from non-small cell lung cancer. Tumori 89: 292-297, 2003. 
8. Girard N, Cottin V, Tronc F, Etienne-Mastroianni B, et al: Chemotherapy is the cornerstone of the combined surgical treatment of lung cancer with synchronous brain metastases. Lung Cancer 53: 51-58, 2006.

9. Paek SH, Audu PB, Sperling MR, Cho J and Andrews DW: Reevaluation of surgery for the treatment of brain metastases: review of 208 patients with single or multiple brain metastases treated at one institution with modern neurosurgical techniques. Neurosurgery 56: 1021-1034, 2005.

10. Li M and Ransohoff RM: Multiple roles of chemokine CXCL12 in the central nervous system: a migration from immunology to neurobiology. Prog Neurobiol 84: 116-131, 2008.

11. Banisadr G, Skrzydelski D, Kitabgi P, Rostène W and Parsadaniantz SM: Highly regionalized distribution of stromal cell-derived factor-1/CXCL12 in adult rat brain: constitutive expression in cholinergic, dopaminergic and vasopressinergic neurons. Eur J Neurosci 18: 1593-1606, 2003.

12. Rempel SA, Dudas S, Ge S and Gutiérrez JA: Identification and localization of the cytokine SDF1 and its receptor, CXC chemokine receptor 4 , to regions of necrosis and angiogenesis in human glioblastoma. Clin Cancer Res 6: 102-111, 2000.

13. Rostasy K, Egles C, Chauhan A, Kneissl M, Bahrani P, Yiannoutsos C, et al: SDF-1alpha is expressed in astrocytes and neurons in the AIDS dementia complex: an in vivo and in vitro study. J Neuropathol Exp Neurol 62: 617-626, 2003.
14. Hill WD, Hess DC, Martin-Studdard A, Carothers JJ, Zheng J, Hale D, et al: SDF-1 (CXCL12) is upregulated in the ischemic penumbra following stroke: association with bone marrow cell homing to injury. J Neuropathol Exp Neurol 63: 84-96, 2004.

15. Miller JT, Bartley JH, Wimborne HJ, Walker AL, Hess DC, Hill WD and Carroll JE: The neuroblast and angioblast chemotaxic factor SDF-1 (CXCL12) expression is briefly up regulated by reactive astrocytes in brain following neonatal hypoxic-ischemic injury. BMC Neurosci 6: 63, 2005.

16. Peng H, Erdmann N, Whitney N, Dou H, Gorantla S, Gendelman HE, et al: HIV-1-infected and/or immune activated macrophages regulate astrocyte SDF-1 production through IL-1beta. Glia 54: 619-629, 2006.

17. Tabatabai G, Frank B, Möhle R, Weller M and Wick W: Irradiation and hypoxia promote homing of haematopoietic progenitor cells towards gliomas by TGF-beta-dependent HIF-1alpha-mediated induction of CXCL12. Brain 129: 2426-2435, 2006.

18. Ceradini DJ, Kulkarni AR, Callaghan MJ, Tepper OM, Bastidas N, Kleinman ME, et al: Progenitor cell trafficking is regulated by hypoxic gradients through HIF-1 induction of SDF-1. Nat Med 10: 858-864, 2004. 